\title{
SIZE EFFECT ON THE ULTIMATE DRYING SHRINKAGE OF CEMENT MORTAR: 1-YEAR EXPERIMENT AND NUMERICAL MODELING
}

\author{
Lenka Dohnalová*, Petr Havlásek, Vít Šmilauer, Pavel Reiterman, \\ VENDULA DAVIDOVÁ
}

\author{
Czech Technical University in Prague, Faculty of Civil Engineering, Department of Mechanics, Thákurova 7, \\ 16629 Prague 6, Czech Republic \\ * corresponding author: lenka.dohnalova@fsv.cvut.cz
}

\begin{abstract}
The magnitude and time evolution of shrinkage are influenced by numerous factors which are implemented in the design codes often in a different way. The time-dependent behavior of concrete in structures sensitive to creep and shrinkage should be verified by means of short-term laboratory measurements. Extrapolation of drying shrinkage from short-term measurements is an illposed problem. The process is extremely slow but can be accelerated by reducing the specimen size. The knowledge of the size-effect on drying shrinkage is a necessity to establish the transition from the laboratory to the structural size. In the literature, the experimental data on such size-effect are insufficient. For this reason a new experiment was developed to study this phenomenon on small-scale specimens made of cement mortar and the results from the first year are summarized in this paper. The measured data are validated by coupled FEM hygro-mechanical simulations.
\end{abstract}

KEYWORDS: Concrete, drying, modeling, shrinkage, size effect.

\section{INTRODUCTION}

Creep and shrinkage of concrete are complex timedependent processes whose physical origin remains still fully unresolved. The importance of both phenomena and the need for their correct prediction grew with the boom of pre-stressed concrete technology which allowed to design slender structures with larger spans and which allowed to utilize concrete potential economically. At the same time, such challenging structures are generally more sensitive to the timedependent deformations in concrete.

The magnitude and time evolution of shrinkage is influenced by numerous factors (ranging from concrete composition to ambient conditions) which are not approached consistently among current prediction models and design codes for concrete. To name a few, the subtle interplay between the development of drying shrinkage and drying creep (investigated e.g. in [1]) can determine whether the durability of concrete structure will be jeopardized by tensile cracking or the shrinkage-induced tensile stresses are relieved sufficiently fast by increased compliance. A disagreement among the different models prevails also in the question of the size-effect on the ultimate value of drying shrinkage [2] which is addressed in this paper.

The resulting discrepancies between the design codes give rise to many uncertainties which can drastically affect the requirements on the design of shrinkage- and creep-sensitive structures. The prediction of the behavior of such structures can be improved by laboratory measurements on specimens prepared from the same concrete composition. This approach can be successfully utilized to specify the estimated concrete creep based on short-time measurements.

However, the direct applicability to drying shrinkage is rather limited because the extrapolation presents an ill-posed problem. The characteristic time and the ultimate shrinkage cannot be determined uniquely until the rate plotted in log-scale significantly decreases [3]. In the case of standard laboratory specimens $70 \times 70 \times 285 \mathrm{~mm}$ or $100 \times$ $100 \times 500 \mathrm{~mm}$, a subtle decrease in shrinkage rate (in log scale) occurs after approx. 100 days while the almost ultimate shrinkage strain is reached after approx. 1000 days of drying [4].

The size-effect on drying shrinkage is in a certain way introduced in majority of design codes or models for time-dependent behavior of concrete. The origin of the size-effect can be sought in nonuniform distribution of relative humidity over the cross-section which gives rise to non-uniform shrinkage strains. The shrinkage strains are internally restrained which produces highly nonlinear distribution of self-equilibrated stresses. Owing to the presence of these stresses in the early stages of drying, concrete is extremely prone to surface cracking. The generated stresses become redistributed as the concrete dries and their magnitude is gradually relieved by concrete creep. This complicated interplay causes that the value of ultimate drying shrinkage decreases with increasing size of the concrete member.

If the size-effect on the ultimate shrinkage is known, the time-demanding experiments can be significantly accelerated when the dimensions of a spec- 

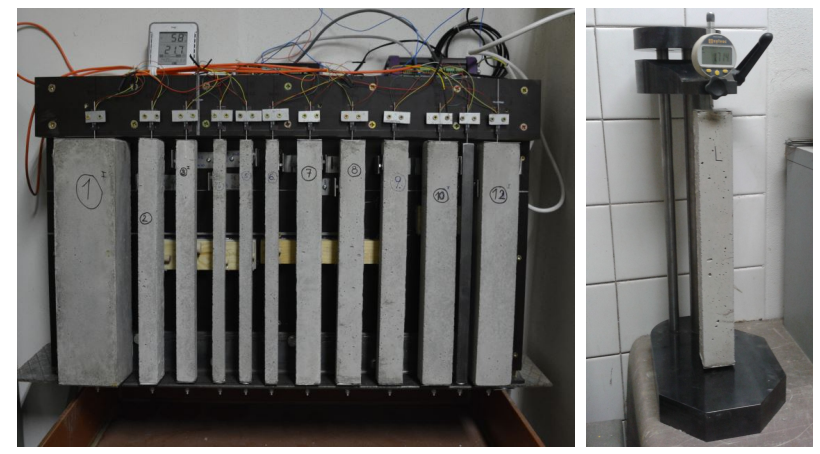

FiguRE 1. Images of the experimental setup: frames for automated (left) and manual (right) readings.

imen become reduced. The rate of drying and thus (drying) shrinkage scales with the square of the effective thickness $D$ defined in B3 model [5] as $2 \mathrm{~V} / \mathrm{S}$ where $V$ is the volume of the specimen and $S$ is the surface exposed to drying. For example in the case of an infinite prism with square cross-section, $D$ coincides with half of its thickness. In this paper, parameter $D$ is used to express the specimen size.

The comprehensive creep and shrinkage database assembled at the Northwestern University [6] comprises a remarkable number of experimental results gathered from the entire world. However, the promising content of this database becomes rather limited when only a specific phenomenon is to be identified. Only a small number of experimental studies examined drying shrinkage of specimens with different sizes prepared from the same concrete mixture and subject to identical conditions. This selection needs to be reduced even further because in many cases the experiment was terminated prematurely before the ultimate shrinkage was reached.

A recent paper by the present authors [2] attempted to evaluate the aforementioned size-effect based on the data available in the literature and the aforementioned database. The experiments unanimously indicate that the ultimate drying shrinkage decreases with specimen size, but this relationship cannot be evaluated more accurately owing to insufficient relevant experimental data.

The lacking data stimulated the present authors to design and execute a new narrow-oriented experiment focused directly on the size-effect on drying shrinkage. This paper presents and summarizes the measured data collected within the first year of the experiment and compares the strain development in time with the results obtained from coupled hygromechanical finite element simulations.

\section{MATERIALS AND METHOdS}

\subsection{EXPERIMENTAL SETUP}

The proposed setup for the shrinkage and moisture loss measurement comprised a large variety of prismatic specimens with uniform length $400 \mathrm{~mm}$ but with cross-section ranging from $20 \times 20 \mathrm{~mm}(D=$
$10 \mathrm{~mm})$ to $100 \times 100 \mathrm{~mm}(D=50 \mathrm{~mm})$. In order to guarantee material homogeneity and to obtain representative data even on the smallest specimens, cement mortar with $2 \mathrm{~mm}$ maximum aggregate size was preferred at the expense of concrete.

The composition of the mortar mix had standard sand-to-cement ratio $3: 1$ by mass. Standard CEN siliceous sand and the blended binder CEM II 32.5 B-S with slag content $29 \%$ were used. The typical water-to-cement ratio was reduced from $w / c=0.5$ to 0.45 to match the behavior of mortar in a typical structural concrete. Compressive strength and Young's modulus measured at the age of 33 days on standard cylinders $100 \times 200 \mathrm{~mm}$ were $33.9 \mathrm{MPa}$ and $40.9 \mathrm{GPa}$, respectively.

The slender prisms were cast into a custom-built steel formwork with plastic bottom. The goal was to resemble curing in sealed conditions. For this reason the top was covered with $1 \mathrm{~mm}$ thick PE sheet followed by a damp cloth. The following day the specimens were transported to a sealed container with $h_{\mathrm{env}}=96 \%$.

The specimens were demolded at the age of 31 days when the drying began. The ends of the prisms were equipped by metal sheets with a central dent to provide suitable surface for the measurement as well as moisture sealing. The reference measurement was done once the glue had hardened.

Altogether 11 mortar prisms with one companion steel specimen for temperature compensation were placed in a special measuring frame, see Fig. 1 left. Each specimen rested on a bolt with round head. Axial shortening was measured at the top using linear transducer with internal spring return MMR1012 (effective stroke $12.7 \mathrm{~mm}$, total resistance $10 \mathrm{k} \Omega$ ). Data logging occurred automatically, the setup did not allow to measure the moisture loss due to drying.

For this reason, a second group consisting of 15 specimens was measured in a more conventional approach using stiff measuring frame equipped with digital indicator Sylvac with $1 \mu \mathrm{m}$ precision, see Fig. 1 right. This setup allowed to determine also the evaporated water which was recorded using precision balance scales; KERN 572-39 (range $4.2 \mathrm{~kg}$, readout $0.01 \mathrm{~g}$ ) and T-scale (range $15 \mathrm{~kg}$, readout $0.1 \mathrm{~g}$ ) for sample with cross-section $100 \times 100 \mathrm{~mm}$. Minimum difference between the coefficient of thermal expansion of steel and mortar allowed relative reading, circumventing temperature compensation.

The experiment was executed in a laboratory without humidity and temperature control. In order to take the inevitable fluctuations into consideration, both temperature and relative humidity were recorded. The frequency corresponded to the automatic and manual measurements.

\subsection{NumericAl MODELING}

The consistency of the measured data was verified using one-way coupled hygro-mechanical simulations 

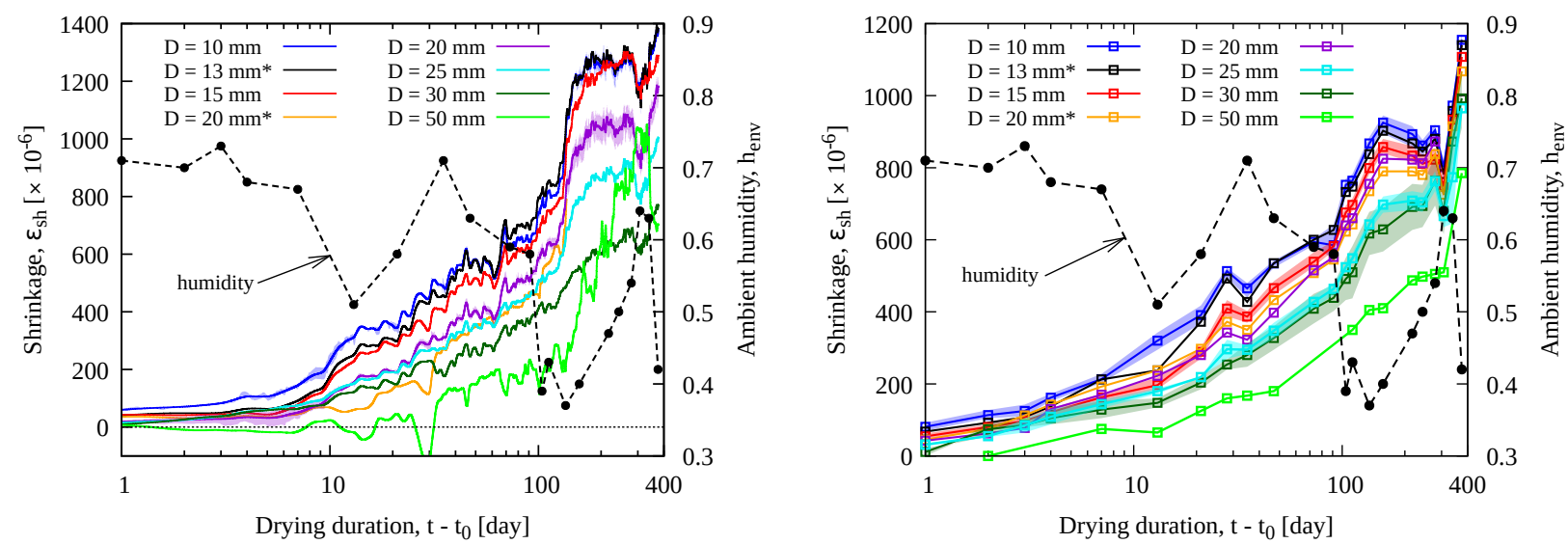

FigURE 2. Evolution of shrinkage measured automatically (left) and manually (right). The shaded area corresponds to the experimental range in the case more identical specimens. Asterisk denotes specimens with rectangular crosssection (square cross-section otherwise).

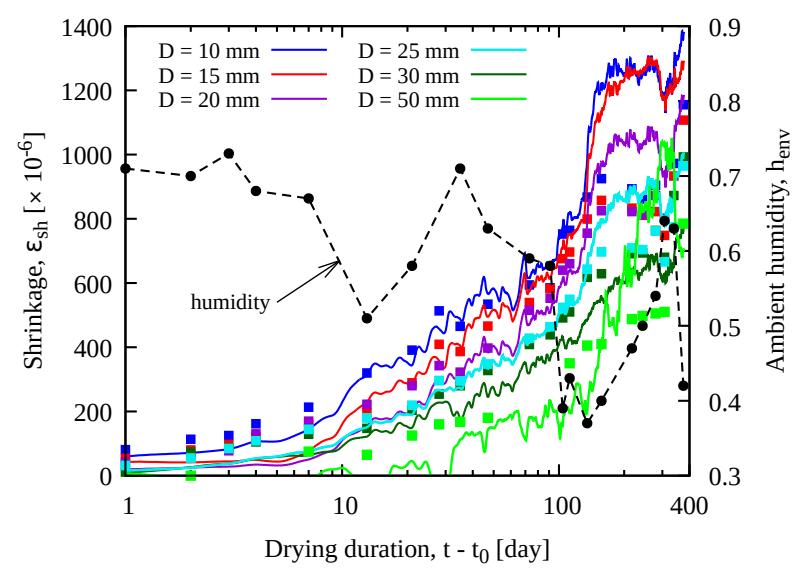

Figure 3. Comparison of the average shrinkage strain measured automatically (solid lines) and manually (symbols). The two techniques gave good agreement during the first 6 months but afterwards started to diverge which was attributed to the deformation of the plywood back of the shrinkage frame for automated measurements.

in open-source finite element package OOFEM [7].

Concrete drying is, in the present study, described by a widely recognized model proposed by Bažant and Najjar [8]. Under certain simplified assumptions, the governing equation for the diffusion of water vapor reads

$$
\frac{\partial h}{\partial t}=\nabla \cdot(C(h) \nabla h)
$$

where $\nabla h$ is the gradient of relative humidity and $C(h)$ is the humidity-dependent diffusivity. This dependence is for the cementitious materials highly nonlinear and can be approximated as

$$
C(h)=C_{1}\left(\alpha_{0}+\frac{1-\alpha_{0}}{1+\left(\frac{1-h}{1-h_{c}}\right)^{n}}\right)
$$

where $C_{1}$ is the maximum diffusivity at $h=1, \alpha_{0}$ determines the ratio between minimum diffusivity at $h=0$ and $C_{1}$, and parameters $h_{c}$ and $n$ describe the relative humidity threshold and the rate of the transition. The ambient relative humidity is prescribed using mixed boundary condition which relates the humidity flux $J_{h}$ with the humidity difference at the boundary via surface factor $f$.

In each time step, the moisture transport is followed by the structural sub-problem which utilizes the computed field of relative humidity. The mechanical problem uses constitutive model based on the Microprestress-solidification (MPS) theory [9]. Under constant temperature and sealed conditions, the MPS model reduces to the B3 model [5].

However, changes in relative humidity give rise not only to volume changes (shrinkage or swelling), but also to additional creep (Pickett effect). The model has been modified [10] to minimize the size-effect on drying creep which is controlled by parameter $k_{3}$. Shrinkage strain and relative humidity are linearly linked via their rates,

$$
\dot{\varepsilon}_{s h}=k_{s h} \dot{h}
$$

where $k_{s h}$ is a material parameter usually treated as a humidity- and age-independent constant. The MPS model is extended with the cohesive crack model and fixed orientation of cracks to consider material softening if the tensile strength is reached. However, the formed cracks do not influence the humidity transport, allowing one-way coupling scheme.

The computational model was calibrated in several subsequent steps. The basic creep compliance, which is in the case of the B3 model described by 4 parameters $q_{1}-q_{4}$, was determined from empirical prediction formulae using compressive strength and concrete composition as input parameters. Afterwards, parameters controlling short-term creep $q_{1}$ and $q_{2}$ were adjusted to match the results of the 1-hour creep experiment. The value of the drying creep parameter, $k_{3}=26$ was identified in the previous analysis of Bryant and Vadhanavikkit experiment 


\begin{tabular}{|c|c|c|c|c|c|c|c|c|c|c|c|}
\hline \multicolumn{3}{|c|}{ B3 model } & \multicolumn{4}{|c|}{ MPS model } & \multicolumn{5}{|c|}{ Moisture diffusivity + mixed b.c. } \\
\hline$q_{1}$ & $\begin{array}{cc}q_{2} & q_{3} \\
{\left[\times 10^{-6} / \mathrm{MPa}\right]}\end{array}$ & $q_{4}$ & $\begin{array}{l}k_{3} \\
{[-]}\end{array}$ & $\begin{array}{c}f_{t} \\
{[\mathrm{MPa}]}\end{array}$ & $\begin{array}{c}G_{f} \\
{[\mathrm{~N} / \mathrm{m}]}\end{array}$ & $\begin{array}{l}k_{s h} \\
{[-]}\end{array}$ & $\begin{array}{c}C_{1} \\
{\left[\mathrm{~mm}^{2} / \text { day }\right]}\end{array}$ & $\begin{array}{l}\alpha_{0} \\
{[-]}\end{array}$ & $\begin{array}{l}h_{c} \\
{[-]}\end{array}$ & $\begin{array}{c}n \\
{[-]}\end{array}$ & $\begin{array}{c}f \\
{[\mathrm{~mm} / \mathrm{day}]}\end{array}$ \\
\hline 15.5 & 1502.08 & 9.41 & 26 & 3.0 & 100 & 0.0016 & 30 & 0.15 & 0.7 & 12 & 0.3 \\
\hline
\end{tabular}

TABLE 1. Summary of material parameters

[11]. The response is only very slightly sensitive to the value of tensile strength $f_{t}$ and fracture energy $G_{f}$, which were set to typical values for concrete (see Tab. 1 which summarizes the values of all basic parameters used in simulations).

The remaining parameter of MPS model $k_{s h}$, and the parameters of the transport model were calibrated to achieve reasonable agreement with the shrinkage results of the performed experiment. The aim was to achieve the best possible match for all prism sizes. The main emphasis was placed on the initial development of shrinkage (approx. first 6 months of drying) when both groups of the specimens show similar development of shrinkage. Later, when there is no longer a match between the specimen groups with a different measurement method, the aim was to achieve the best possible match with the results of the manually measured prisms.

For the purpose of identification of material parameters, the evolution of ambient relative humidity was defined by a piecewise-linear function according to the measured values. In addition, the analysis was repeated for a partially adjusted development of ambient humidity (beginning according to the experiment, after 91 days of drying assumed humidity fixed at $h_{\mathrm{env}}=60 \%$ ) achieve final values of shrinkage and to demonstrate more clearly the size-effect on drying shrinkage predicted by the model.

\section{Results AND DIsCUSSION}

The development of shrinkage in time is for both automated and manual measurement techniques shown in Fig. 2. In the case of more identical specimens, the mean response is shown in solid line while the shaded area corresponds to the measured experimental range. The obvious fluctuations in shrinkage strain are caused by oscillating value of ambient relative humidity which is shown in dashed black line.

The average responses obtained with the two techniques are compared in Fig. 3. Except of the largest specimen, the measurements demonstrate very good agreement during the first 6 months of drying. Sudden decrease in relative humidity from $60 \%$ to $40 \%$, which occurred afterwards, caused rapid acceleration of drying accompanied by increase in shrinkage rate.

This decrease also led to separation of the experimental trends determined by the two methods; in all cases the shrinkage strain measured automatically exceeded the manual readings. The reason for this was not clarified even by thorough analysis of the measured data. Still, the most probable cause seems to

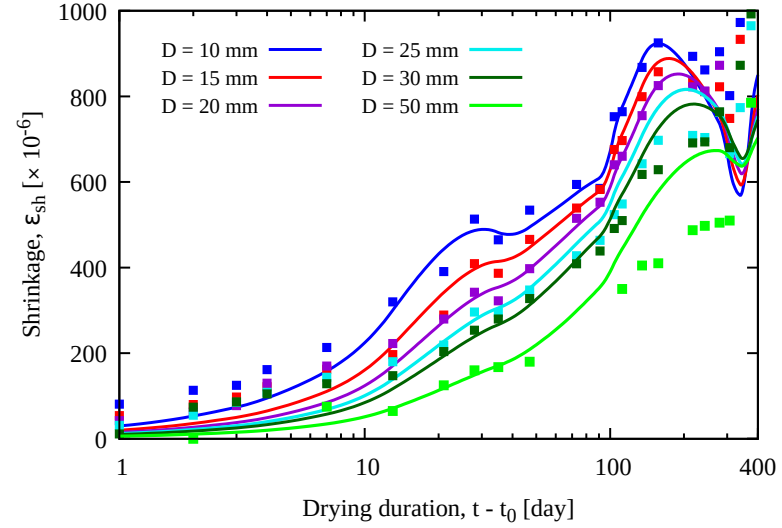

FiguRE 4. Comparison of the experimental measurements (symbols) with the results obtained from FEM simulations (solid lines).

be connected to the frame for automated measurement. The measuring frame was attempted to be sufficiently stiff for the current measurement. Since the frame serves only as a support for the specimens and is loaded merely by their self weight, the selected material was $26 \mathrm{~mm}$ thick plywood for concrete molds. However, this material turned out to be insufficiently stable under variable relative humidity of the ambient environment since non-negligible volume changes were observed. These changes could not be completely eliminated by incorporating the measurement of the steel standard primarily intended for temperature compensation. The difference between the shrinkage of geometrically identical specimens determined by the automated and manual measurement technique was not uniform and was found to be, in a certain way, influenced by their position, namely by the distance from the largest specimen placed at one end (see Fig. 1, the weight of the prism labeled " 1 " reached almost $10 \mathrm{~kg}$ ). The joint effect of these two factors seems to be the most probable explanation.

Therefore, the data from the manual measurements are preferred in the later stage of the experiment (solid steel structure of the measuring frame insensitive to level of relative humidity) despite the intervals between the individual measurements were gradually increasing.

As was stated above, the manual measurements allowed to determine not only the axial shrinkage but also the evolution of weight loss due to drying. To the best knowledge of the authors, such data are not available in this extent in any of the preceding experiments related to the size effect on drying shrinkage. 

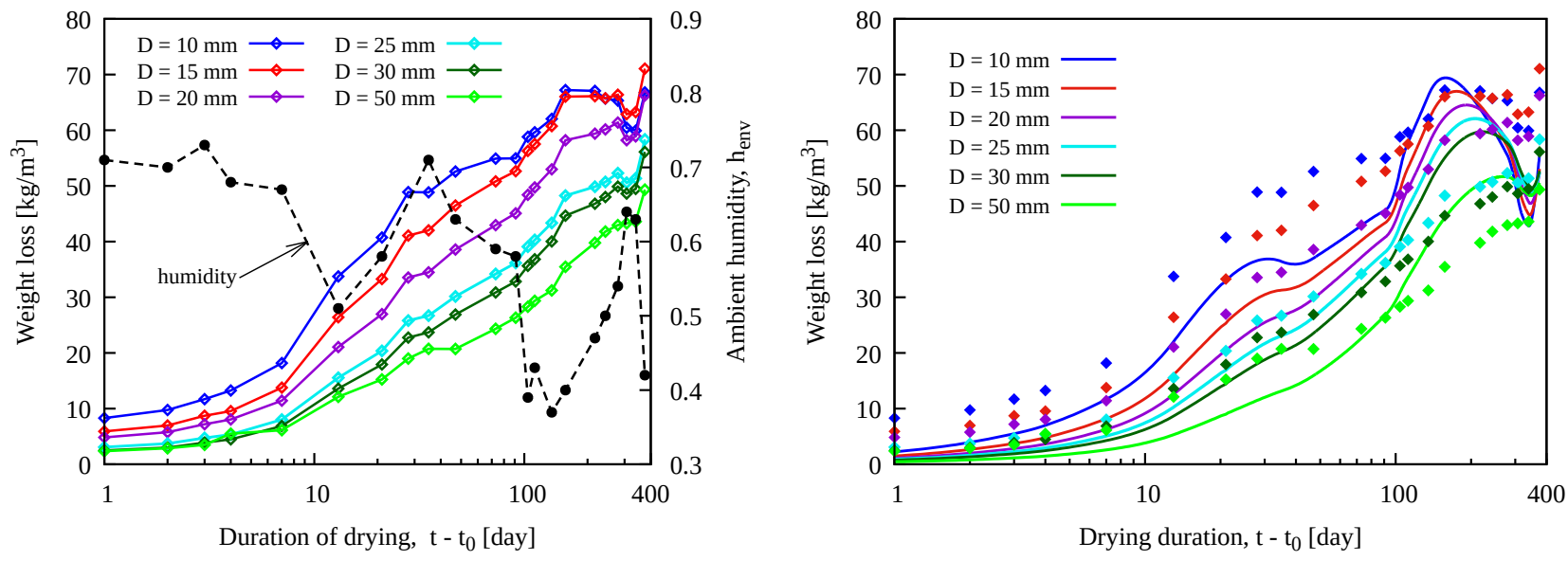

FiguRE 5. Experimentally measured moisture loss (left) and its comparison to FEM analysis (right). After 100 days of drying the model exhibits excessive sensitivity to changes in $h_{\mathrm{env}}$.
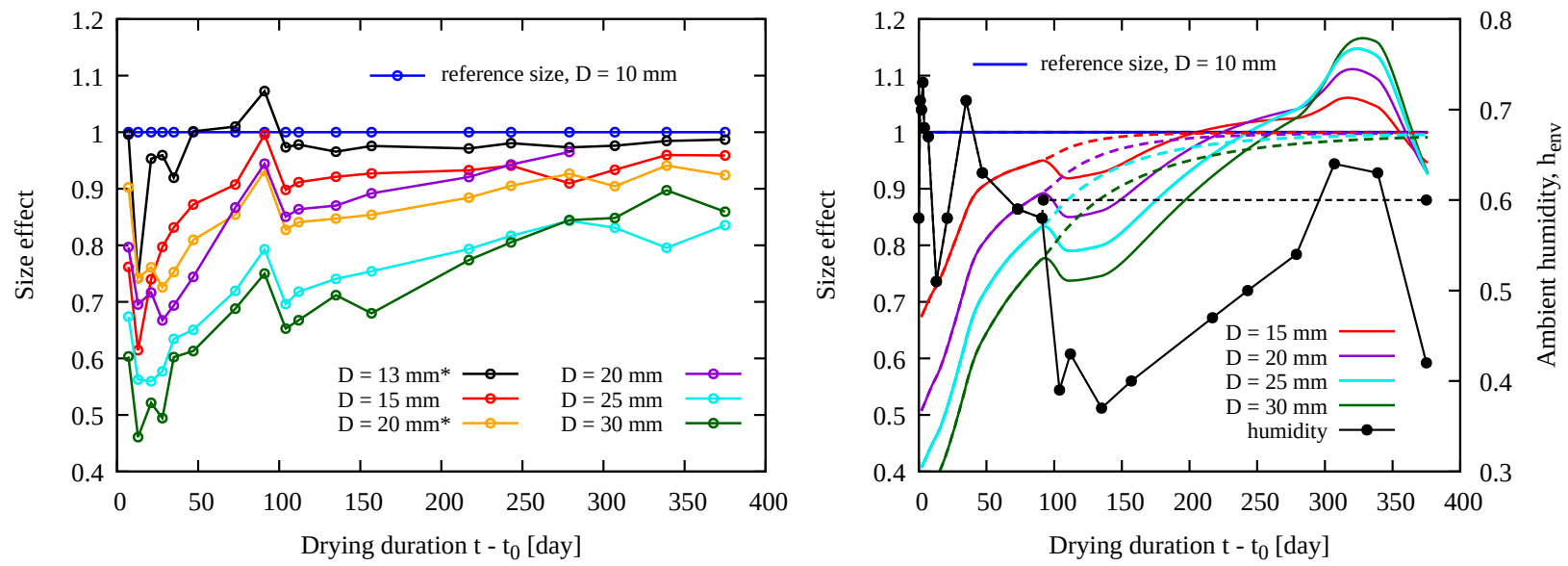

Figure 6. Time development of the experimentally measured (left) and computed (right) size-effect on drying shrinkage. The data points correspond to the average values obtained from manual measurement. The ambient relative humidity recorded in the experiment resulted into the FEM response shown in solid lines while the modified history of humidity ( $h_{\mathrm{env}}=60 \%$ since 91 days of drying) led to the results shown in dashed lines.

Yet such data provide better understanding of the time development of the drying process. The measured evolution of moisture loss normalized per volume of the specimen is shown in the left part of Fig. 5. The sensitivity to changes in ambient relative humidity which occurred in the later stage of drying is in the case of moisture loss not so pronounced as in the case of drying shrinkage.

In the finite element simulations the driving force for the moisture transport is the relative humidity, therefore the moisture loss needs to be backcalculated. The value of moisture capacity (slope of the desorption isotherm, for simplicity assumed as linear) which gave the best agreement with the experimental data as shown in right part of Fig. 5 was $k=120 \mathrm{~kg} / \mathrm{m}^{3}$. In the simulations, the development of ambient relative humidity corresponded with the experimental measurements. The agreement with the experimental data is satisfactory only in the first 100 days of drying; afterwards, the model over- estimates the rate of the weight loss or gain caused by changes in $h_{\text {env }}$. The discrepancy in the initial value of weight loss stems from the linear desorption isotherm which is not realistic in the case of high relative humidity.

The ongoing changes in $h_{\text {env }}$ did not allow the specimens to reach their ultimate value of drying shrinkage. For this reason the size effect on drying shrinkage is here evaluated within the entire duration of the experiment and is related to the thinnest specimen with cross-section $20 \times 20 \mathrm{~mm}(D=10 \mathrm{~mm})$. Naturally, the strongest size-effect can be observed shortly after the onset of drying when the difference between the moisture loss of specimens with different size is most remarkable. On the other hand with the increasing duration the size-effect is slowly diminishing until the equilibrium with the ambient conditions is reached.

The expected evolution of the size-effect on drying shrinkage is confirmed by the experimental measure- 
ments as shown in the left part of Fig. 6. Even though the ultimate values of drying shrinkage have not been reach so far, the size-effect has become almost stable and there is no indication that is should vanish completely. The processed data are in correct order, the size-effect increases with size. With respect to the reference specimen $(D=10 \mathrm{~mm})$ the size effect is within $20 \%$ of that value.

As was shown in Fig. 4, the experimentally measured evolution of shrinkage was captured by the numerical model very realistically up to $\approx 180$ days of drying. However, the mismatch beyond this time causes that the numerically computed size-effect significantly deviates from the experiment as demonstrated by the smooth solid lines in the right part of Fig. 6. In order to arrive at equilibrated values of drying shrinkage, the simulation was repeated with a modified history of $h_{\mathrm{env}}$, which was fixed at $60 \%$ after 91 days of drying. Under such conditions the predicted size-effect becomes almost negligible within one year of drying.

\section{Conclusions}

This paper analyzed and presented the results from the first year of a pilot experiment developed to investigate the size-effect on drying shrinkage. The primary motivation of this research was to verify whether the shrinkage measurements could be accelerated using smaller specimens than is the current practice.

The findings can be summarized as follows.

- The difficulties encountered in the first run of the experiment provided the research team with a valuable feedback for its future improvement. The experimental technique itself is feasible, cheap and capable to deliver good-quality data. A stiff steel frame, automatic monitoring of ambient conditions are a necessity, while the currently used monitoring of moisture loss is sufficient.

- The measured data were in expected order and exhibited very little scatter among specimens with the same dimensions. This indicates good homogeneity of the material in the specimens despite their small dimensions. Moreover, the consistency of the data was verified by a computational model and the agreement was promising.

- The size-effect on drying shrinkage cannot be properly evaluated unless the experiment is conducted at constant relative humidity, which was not the present case. Despite this deficiency, in the currently examined size range a non-negligible sizeeffect on drying shrinkage was registered.
- The constitutive model used in structural analysis needs to be improved as it strongly underestimates the size-effect on drying shrinkage.

\section{ACKNOWLEDGEMENTS}

The authors gratefully acknowledge financial support from the Czech Science Foundation (GA ČR), project number 19-20666S.

\section{REFERENCES}

[1] L. Dohnalová, P. Havlásek. Comparison of drying shrinkage and drying creep kinetics in concrete. Acta Polytechnica CTU Proceedings 15:12-19, 2018.

[2] L. Dohnalová, P. Havlásek. Size effect on the ultimate drying shrinkage of concrete - experimental evidence and engineering practice. Acta Polytechnica CTU Proceedings 26:13-18, 2020.

[3] Z. Bažant, M. Jirásek. Creep and Hygrothermal Effects in Concrete Structures. Springer Netherlands, 2018. DOI:10.1007/978-94-024-1138-6.

[4] C. Aguilar. Estudio del comportamiento y desarrollo de una metodologia de prediccion de la retraccion hidraulica de hormigones, PhD. Thesis (in Spanish). Pontificia Universidad Catolica de Chile. Escuela de Ingenieria, 2005.

[5] Z. Bažant, S. Baweja. Creep and shrinkage prediction model for analysis and design of concrete structures: Model B3. Adam Neville Symposium: Creep and Shrinkage - Structural Design Effects 2000.

[6] M. Hubler, R. Wendner, Z. Bažant. Comprehensive database for concrete creep and shrinkage: analysis and recommendations for testing and recording. ACI 112:547-558, 2015.

[7] B. Patzák. OOFEM home page, 2000. Http://www.oofem.org.

[8] Z. P. Bažant, L. J. Najjar. Nonlinear water diffusion in nonsaturated concrete. Materials and Structures 5:3-20, 1972. DOI:10.1007/BF02479073.

[9] Z. P. Bažant, A. P. Hauggaard, S. Baweja, F. J. Ulm. Microprestress solidification theory for concrete creep. I: Aging and drying effects. Journal of Engineering Mechanics 123:1188-1194, 1997.

[10] Z. Bažant, P. Havlásek, M. Jirásek. Microprestress-solidification theory: Modeling of size effect on drying creep. In N. Bicanic, H. Mang, G. Meschke, R. de Borst (eds.), Computational Modelling of Concrete Structures, pp. 749-758. CRC Press/Balkema, EH Leiden, The Netherlands, 2014.

[11] A. H. Bryant, C. Vadhanavikkit. Creep, shrinkage-size, and age at loading effects. $A C I$ Materials Journal 84:117-123, 1987. 